\title{
Beurteilung der Weiterbildung durch Fachärztinnen und Fachärzte
}

\author{
Michael Siegrist ${ }^{a}$, Bernadette Sütterlin ${ }^{b}$, Lea Christina Burgermeister ${ }^{c}$, Werner Bauer $^{d}$ \\ ${ }^{\text {a }}$ Prof. Dr. phil., Professor für Consumer Behavior an der ETH Zürich; ${ }^{b}$ Dr. sc., Senior Researcher; ${ }^{c}$ Lic. phil., wissenschaftliche Mitarbeiterin; \\ d Dr. med., Präsident SIWF
}

Werden in der Weiterbildung zum Facharzt diejenigen Themenbereiche, die für die spätere berufliche Tätigkeit relevant sind, ausreichend abgedeckt? Und wie gut werden die entsprechenden Kompetenzen vermittelt? Dies sind entscheidende Fragen für die Qualität der Facharztweiterbildung in der Schweiz.

Die jährliche Befragung der Ärztinnen und Ärzte in Weiterbildung liefert wichtige Informationen, die als Grundlagen für Verbesserungen dienen können [1]. Sie lässt aber keine Schlussfolgerungen darüber $\mathrm{zu}$, ob während der Weiterbildung auch alle Kenntnisse und Fähigkeiten ausreichend vermittelt werden, die für die spätere praktische Tätigkeit und auch für die berufliche Weiterentwicklung wichtig sind.

Eine standardisierte Befragung, in der Ärztinnen und Ärzte in Weiterbildung nach Abschluss ihrer Weiterbildung beurteilen, wie gut sie auf die praktische Arbeit vorbereitet wurden, ermöglicht es, die Weiterbildungsgänge im Hinblick auf diese Fragestellung zu evaluieren $[2,3]$. Für die Schweiz gab es bisher jedoch keine Daten darüber, wie die praktizierenden Ärztinnen und Ärzte ihre Weiterbildung und die Facharztprüfung beurteilen. Um diese Wissenslücke zu füllen, wurden die Facharztabsolventinnen und -absolventen, die 2009 bis 2011 einen Schweizer Facharzttitel erworben hatten, zu ihrer Beurteilung der Weiterbildung befragt. Im Folgenden werden die für die Befragung verwendete Methode und der Fragebogen kurz beschrieben. Anschliessend werden die wichtigsten Resultate der Befragung dargestellt und diskutiert. Da es sehr grosse fachspezifische Unterschiede in den Erwartungen und den gelehrten Kompetenzen gibt [4], werden die meisten Ergebnisse nach den wichtigsten Fachrichtungen aufgegliedert präsentiert.

\section{Methode}

\section{Stichprobe}

Die Fragebogen wurden an 3367 Ärztinnen und Ärzte verschickt, welche innerhalb der letzten fünf Jahre ihren Facharzttitel erhalten hatten. Die Daten wurden
Ende 2013 bis Anfang 2014 erhoben. Die Rücklaufquote betrug 48\% ( $\mathrm{N}=1623)$. Von den retournierten Fragebogen wurden $47 \%$ von Fachärztinnen und 53\% von Fachärzten ausgefüllt. In $32 \%$ der Fälle besassen die Befragten mehr als einen Facharzttitel und etwa die Hälfte (51\%) hatte eine Kaderposition inne. Die Mehrheit der Befragten war als spezialisierte Fachärztin oder als spezialisierter Facharzt tätig (74\%).

\section{Fragebogen}

Der Fragebogen beinhaltete 113 Fragen und war in drei Sprachversionen verfügbar. Im Fragebogen wurde erfasst, wie gut unterschiedliche Kompetenzen in der Weiterbildung vermittelt wurden und als wie wichtig diese Kompetenzen für die tägliche Arbeit beurteilt werden. Weiter wurden Fragen zur allgemeinen Zufriedenheit mit der Weiterbildung, zu den gesetzten Lernzielen und den zu erfüllenden Operationskatalogen sowie zur Facharztprüfung gestellt. Bei den Fachkompetenzen mussten die Befragten zwei Beurteilungen vornehmen. Bei der ersten Beurteilung zur Frage, wie gut die jeweiligen Fachkompetenzen vermittelt wurden, wurden die Teilnehmer gebeten, auf einer Antwortskala von 1 «ungenügend weitergebildet» bis 6 «ausgezeichnet weitergebildet» ihre Einschätzung abzugeben. Bei der zweiten Beurteilung ging es um die Wichtigkeit der verschiedenen Fachkompetenzen für die praktische Tätigkeit der Befragten. Für die Beurteilung standen die Antwortmöglichkeiten 1 "gar nicht wichtig» bis 6 «sehr wichtig» zur Auswahl. Spielt eine Kompetenz in der jeweiligen Fachrichtung keine Rolle, so konnte die Antwortmöglichkeit «trifft nicht auf meine Weiterbildung zu» bzw. "trifft nicht auf meine Tätigkeit zu» angekreuzt werden. Diese Werte flossen nicht in die Berechnungen ein. Die Fragen zum Lernzielkatalog sowie zur Zufriedenheit mit der Weiterbildung zum Facharzt / zur Fachärztin wurden in Form von Aussagen formuliert. Die Teilnehmer wurden gebeten, anzugeben, inwiefern die Aussagen aus ihrer Sicht zutreffen. Dabei standen sechs Antwortkategorien von 1 "trifft überhaupt nicht zu» bis 6 «trifft voll und ganz zu» zur Auswahl. 


\section{Ergebnisse}

\section{Vermittlung der relevanten Fachkompetenzen}

Für die Auswertungen zur Qualität der Weiterbildung in den einzelnen Fachkompetenzen und zu deren Wichtigkeit für die Ausübung ihrer Tätigkeit als Facharzt und Fachärztin wurden nur die Daten jener Befragten berücksichtigt, die nach dem Erwerb ihres Facharzttitels mindestens drei Monate berufstätig waren $(\mathrm{N}=1596)$. Die Ergebnisse für die sieben Fachrichtungen mit den meisten Weiterbildungsstellen sind in Abbildung 1 dargestellt. Um die Interpretation zu erleichtern, wurden die Fachkompetenzen nach der Differenz zwischen der Einschätzung der Qualität der Weiterbildung und der Wichtigkeit für die praktische Tätigkeit geordnet.

\section{Anästhesiologie}

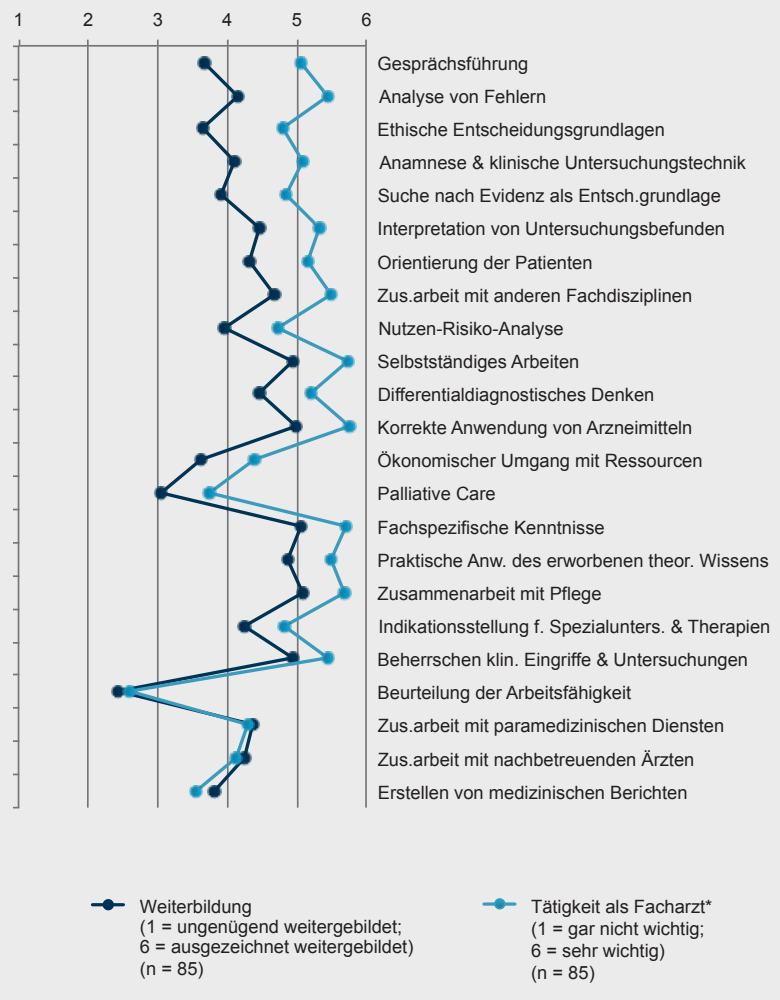

Abbildung 1: Einschätzung der vermittelten Fachkompetenzen im Hinblick auf die Fragen «Wie gut wurden Sie in Ihrer Weiterbildung zum Facharzt in den folgenden Kompetenzen, Fähigkeiten beziehungsweise Kenntnissen weitergebildet?» und «Wie wichtig sind/waren die folgenden Kompetenzen, Fähigkeiten beziehungsweise Kenntnisse für die Ausübung Ihrer Tätigkeit als Facharzt?», dargestellt für die sieben Fachrichtungen mit den meisten Weiterbildungsstellen. Sortiert nach der Grösse der Differenz zwischen Qualität der Weiterbildung und Wichtigkeit für die praktische Tätigkeit. (Abbildung über zwei Seiten)

* Für die Auswertungen wurden nur die Antworten von Befragten berücksichtigt, die nach Erwerb des Facharzttitels mindestens drei Monate berufstätig waren $(\mathrm{N}=1596)$.

\section{Allgemeine Innere Medizin}

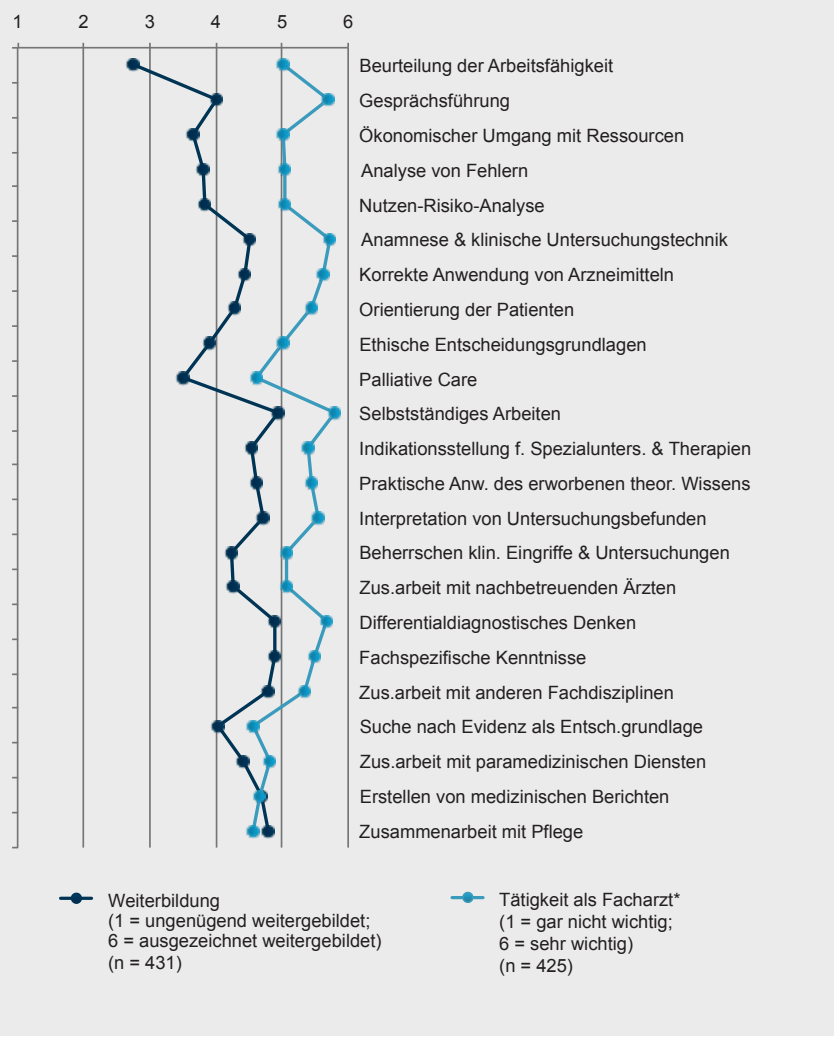

\section{Gynäkologie}

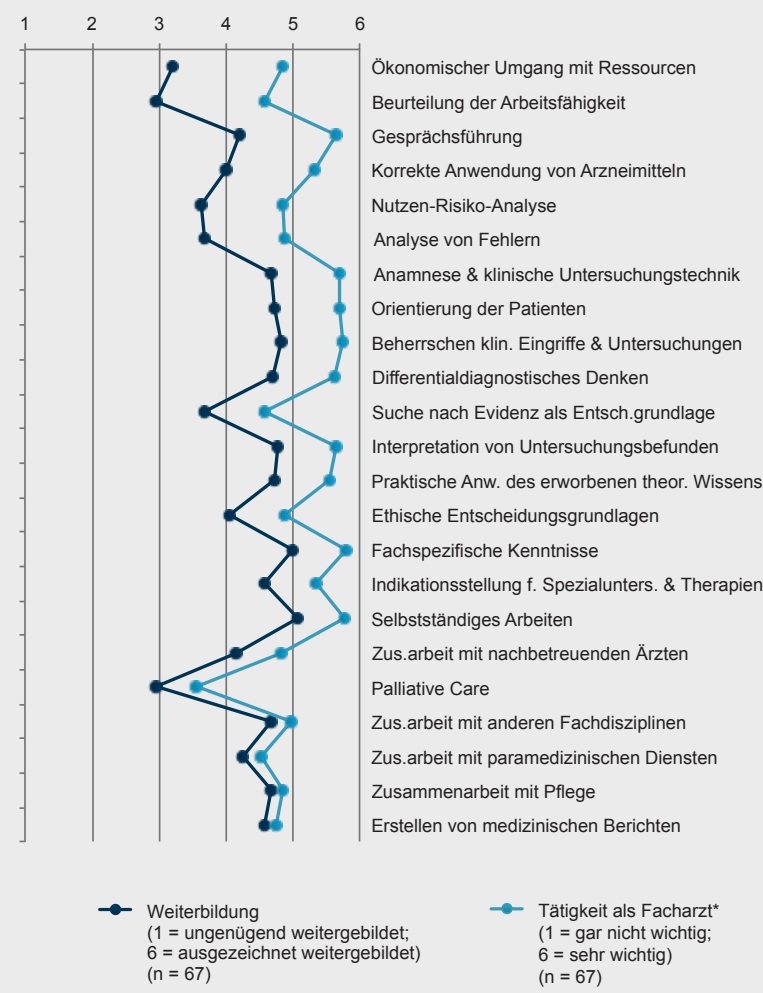




\section{Chirurgie}

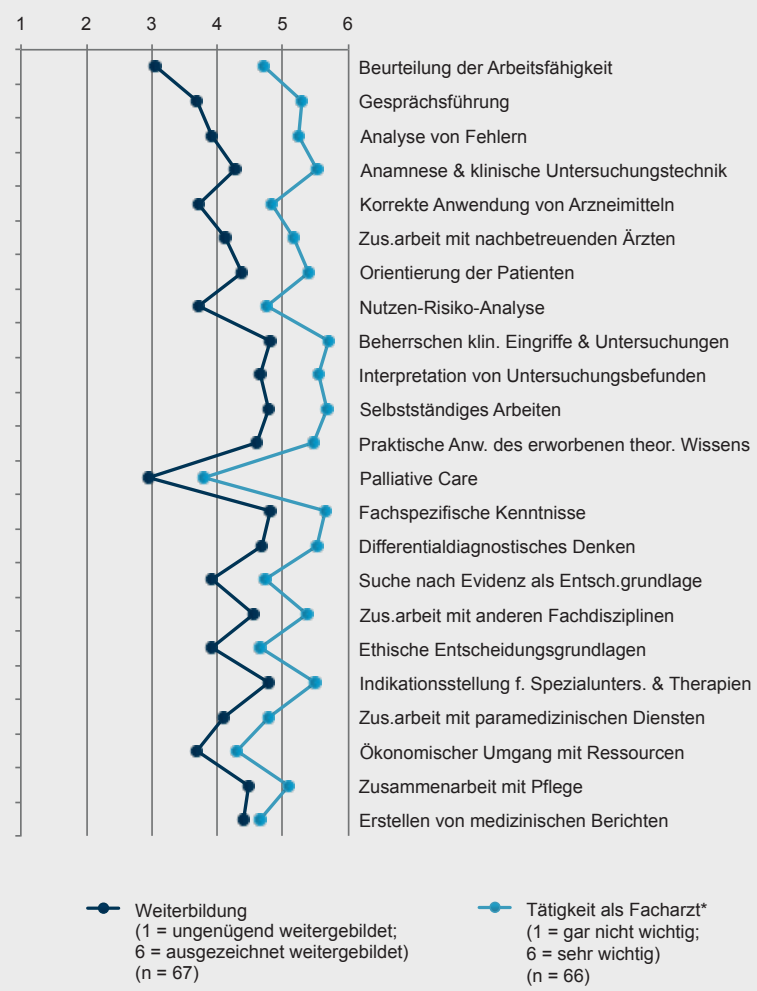

\section{Psychiatrie}

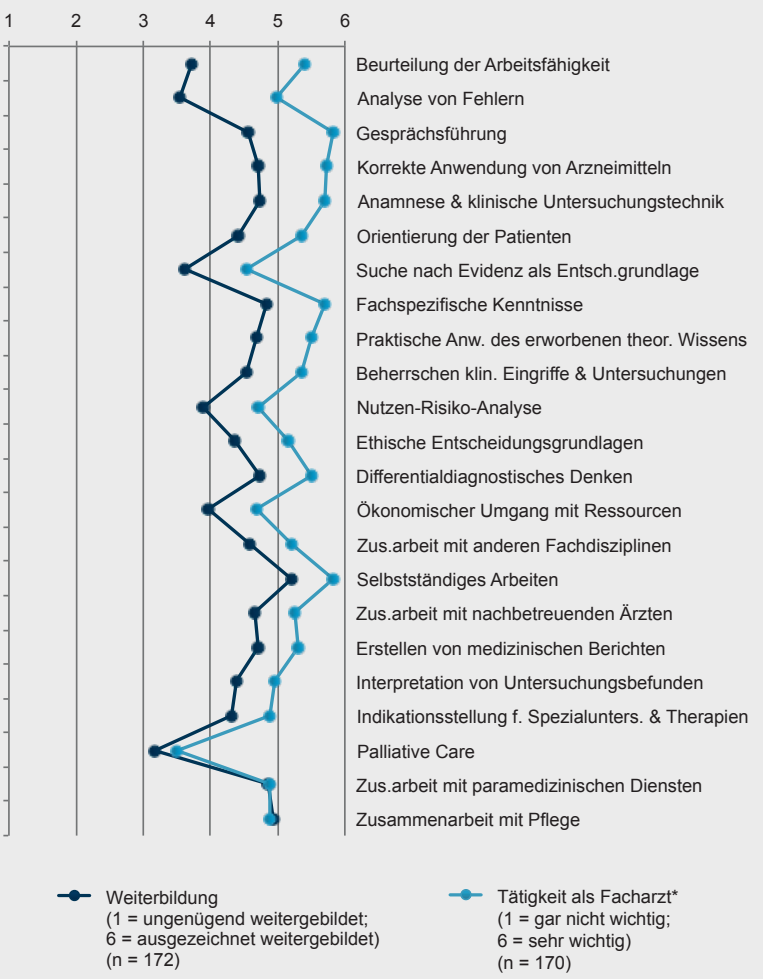

\section{Kinder- und Jugendmedizin}

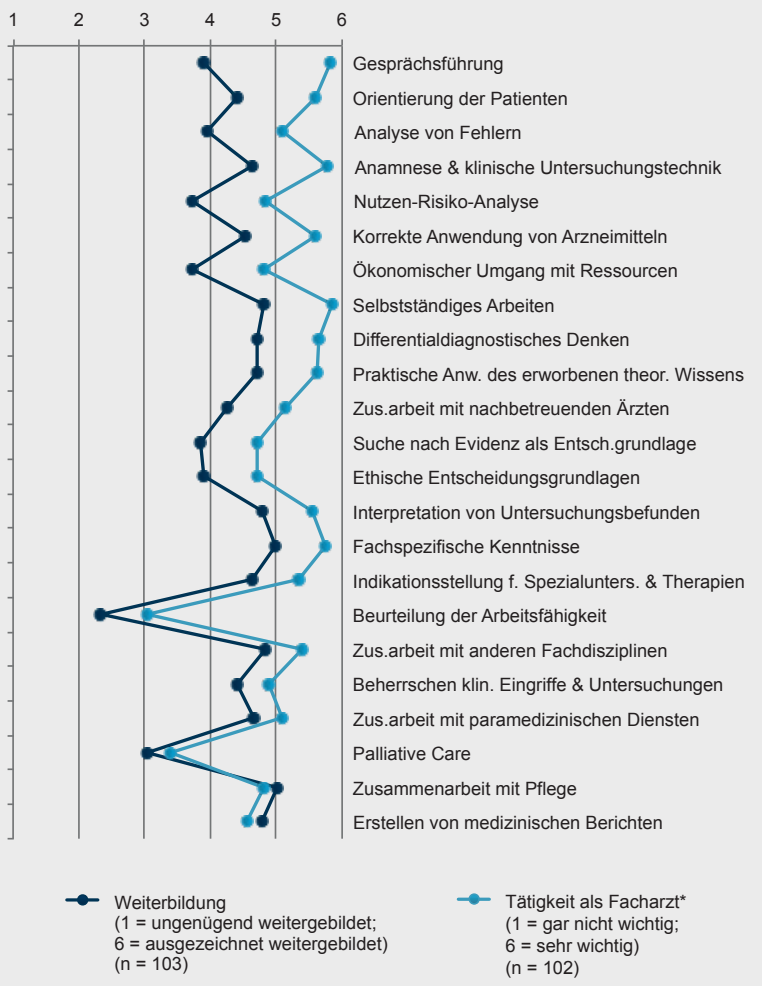

\section{Orthopädische Chirurgie}

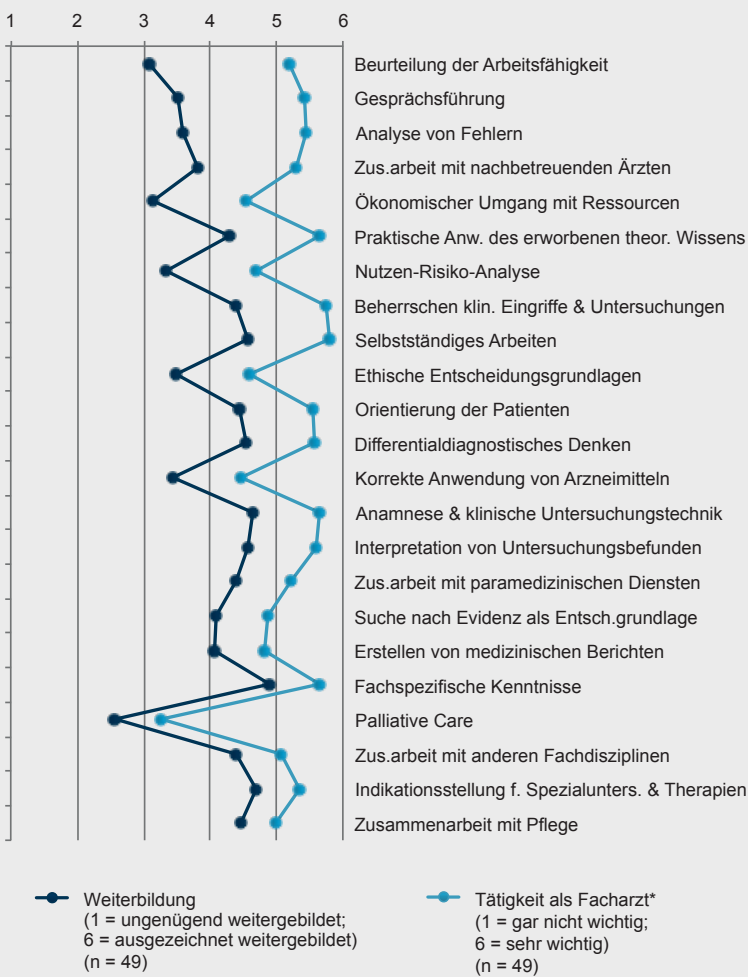


Werden alle Fachrichtungen zusammen analysiert, so sind bei den Kompetenzen «Beurteilung der Arbeitsfähigkeit», "Gesprächsführung» sowie "Analyse von Fehlern" die grössten Differenzen zwischen der Qualität der Weiterbildung und der Wichtigkeit für die Tätigkeit als Facharzt zu beobachten. Der Abbildung 1 kann entnommen werden, dass die Beurteilung der Arbeitsfähigkeit, ausser in der Anästhesiologie und der Kinder- und Jugendmedizin, als wichtig bis sehr wichtig eingestuft wird. Die Vermittlung dieser Kompetenz wird aber in allen fünf Fachrichtungen als ungenügend oder knapp genügend beurteilt. Die Gesprächsführung wird von allen sieben Fachrichtungen als wichtig bis sehr wichtig beurteilt. Die Qualität der Weiterbildung in der Gesprächsführung wird jedoch schlechter beurteilt als bei anderen Kompetenzen, die in der Praxis von vergleichbarer Relevanz sind. Es gibt aber auch klare fachspezifische Diskrepanzen zwischen den beiden Beurteilungen der Kompetenzen. In der Gynäkologie ist das empfundene Defizit beim «ökonomischen Umgang mit Ressourcen" und in der Orthopädischen Chirurgie das gleiche Ergebnis für «Zusammenarbeit mit nachbetreuenden Ärzten» augenfällig.

\section{Erfüllung der Lernziel- und Operationskataloge}

Die Lernziele im Zusammenhang mit den sieben Rollenfunktionen des Arztes (nach CanMEDS) sind lediglich $10 \%$ der Befragten bekannt. Die fachspezifischen Lernziele der Weiterbildungsprogramme scheinen bekannter zu sein. Die Befragten mussten diese rückblickend beurteilen (Tab. 1). Die Antworten wur- den für eine übersichtlichere Darstellung in drei Kategorien zusammengefasst: trifft nicht zu (1-2), unentschieden (3-4), trifft zu (5-6).

Die Fachärztinnen und Fachärzte beurteilen die Lernziele als eine gute Grundlage für die medizinisch selbständige Berufstätigkeit. Aus Sicht der Befragten, insbesondere derjenigen aus der Chirurgie sowie der Orthopädischen Chirurgie und Traumatologie des Bewegungsapparates, gibt es allerdings überflüssige Lernziele im Weiterbildungsprogramm. Ein relativ grosser Teil der Befragten ist aber auch der Meinung, dass wichtige Lernziele im Weiterbildungsprogramm fehlen. Besonders stark scheint diese Meinung unter Kinder-und Jugendmedizinern und Chirurgen vertreten zu sein.

Wie gut lassen sich die in den Weiterbildungsprogrammen enthaltenen Lernziele innerhalb der vorgegebenen Zeit erreichen? In der Anästhesiologie und in der Kinder- und Jugendmedizin scheint dies kein Problem zu sein. Anders ist die Situation dagegen in der Gynäkologie und Geburtshilfe sowie der Orthopädischen Chirurgie und Traumatologie des Bewegungsapparates. In diesen beiden Fachrichtungen ist jeder vierte Befragte der Ansicht, dass die Lernziele nicht in der vorgegebenen Zeit erreicht werden können.

Von den befragten Ärztinnen und Ärzten besitzen 20\% ( $n=322$ ) einen Titel mit operativer Tätigkeit. Diese schätzen zu einem grossen Teil die Anzahl der im Operationskatalog verlangten Eingriffe als angemessen ein (79\%). Nur 9\% halten die Anzahl der verlangten Eingriffe für zu hoch und $12 \%$ schätzen sie als zu tief ein.

Tabelle 1: Beurteilung der Lernziele des Weiterbildungsprogramms

\begin{tabular}{|c|c|c|c|c|c|c|c|c|c|c|c|c|}
\hline & \multicolumn{3}{|c|}{$\begin{array}{l}\text { Die Lernziele (Inhalt der Wei- } \\
\text { terbildung, vgl. Punkt } 3 \text { des } \\
\text { Weiterbildungsprogramms) } \\
\text { bilden eine gute Grundlage } \\
\text { für eine sichere medizinisch- } \\
\text { selbständige Berufstätigkeit. }\end{array}$} & \multicolumn{3}{|c|}{$\begin{array}{l}\text { Im Weiterbildungsprogramm } \\
\text { hat es überflüssige Lernziele. }\end{array}$} & \multicolumn{3}{|c|}{$\begin{array}{l}\text { Im Weiterbildungsprogramm } \\
\text { fehlen wichtige Lernziele. }\end{array}$} & \multicolumn{3}{|c|}{$\begin{array}{l}\text { Es ist möglich, innerhalb der } \\
\text { im Weiterbildungsprogramm } \\
\text { vorgegebenen Zeit die Lern- } \\
\text { ziele zu erreichen. }\end{array}$} \\
\hline & $\begin{array}{l}\text { trifft } \\
\text { nicht zu }\end{array}$ & $\begin{array}{l}\text { unent- } \\
\text { schieden }\end{array}$ & $\begin{array}{l}\text { trifft } \\
\text { zu }\end{array}$ & $\begin{array}{l}\text { trifft } \\
\text { nicht zu }\end{array}$ & $\begin{array}{l}\text { unent- } \\
\text { schieden }\end{array}$ & $\begin{array}{l}\text { trifft } \\
\mathrm{zu}\end{array}$ & $\begin{array}{l}\text { trifft } \\
\text { nicht zu }\end{array}$ & $\begin{array}{l}\text { unent- } \\
\text { schieden }\end{array}$ & $\begin{array}{l}\text { trifft } \\
\mathrm{zu}\end{array}$ & $\begin{array}{l}\text { trifft } \\
\text { nicht zu }\end{array}$ & $\begin{array}{l}\text { unent- } \\
\text { schieden }\end{array}$ & $\begin{array}{l}\text { trifft } \\
\text { zu }\end{array}$ \\
\hline Gesamt (N = 1623) & $4 \%$ & $48 \%$ & $48 \%$ & $37 \%$ & $40 \%$ & $23 \%$ & $32 \%$ & $40 \%$ & $28 \%$ & $9 \%$ & $30 \%$ & $61 \%$ \\
\hline $\begin{array}{l}\text { Allgemeine } \\
\text { Innere Medizin ( }=431 \text { ) }\end{array}$ & $5 \%$ & $53 \%$ & $42 \%$ & $34 \%$ & $43 \%$ & $23 \%$ & $29 \%$ & $38 \%$ & $33 \%$ & $4 \%$ & $34 \%$ & $61 \%$ \\
\hline Anästhesiologie ( $n=85$ ) & $4 \%$ & $53 \%$ & $43 \%$ & $39 \%$ & $40 \%$ & $21 \%$ & $30 \%$ & $40 \%$ & $30 \%$ & $3 \%$ & $25 \%$ & $73 \%$ \\
\hline Chirurgie $(n=67)$ & $2 \%$ & $57 \%$ & $42 \%$ & $19 \%$ & $42 \%$ & $39 \%$ & $22 \%$ & $40 \%$ & $37 \%$ & $13 \%$ & $30 \%$ & $57 \%$ \\
\hline Gynäkologie ( $n=67$ ) & $3 \%$ & $39 \%$ & $58 \%$ & $43 \%$ & $30 \%$ & $27 \%$ & $29 \%$ & $42 \%$ & $29 \%$ & $27 \%$ & $32 \%$ & $41 \%$ \\
\hline $\begin{array}{l}\text { Kinder- und } \\
\text { Jugendmedizin }(n=103)\end{array}$ & $3 \%$ & $44 \%$ & $53 \%$ & $36 \%$ & $42 \%$ & $22 \%$ & $22 \%$ & $38 \%$ & $41 \%$ & $3 \%$ & $17 \%$ & $80 \%$ \\
\hline Psychiatrie (n = 172) & $5 \%$ & $51 \%$ & $44 \%$ & $41 \%$ & $39 \%$ & $20 \%$ & $30 \%$ & $41 \%$ & $29 \%$ & $16 \%$ & $36 \%$ & $48 \%$ \\
\hline $\begin{array}{l}\text { Orthopädische } \\
\text { Chirurgie }(n=49)\end{array}$ & $6 \%$ & $55 \%$ & $38 \%$ & $25 \%$ & $29 \%$ & $46 \%$ & $21 \%$ & $46 \%$ & $33 \%$ & $23 \%$ & $27 \%$ & $50 \%$ \\
\hline
\end{tabular}

Anmerkungen: Frage: «Inwiefern treffen die folgenden Aussagen zu den Lernzielen des Weiterbildungsprogramms, nach dem Sie Ihre Weiterbildung absolviert haben, zu?» Die Antwortskala reichte von 1 «trifft überhaupt nicht zu» bis 6 «trifft voll und ganz zu». Für die Auswertungen wurden die Antworten wie folgt zusammengefasst: 1 , 2 = «trifft nicht zu»; 3, $4=$ "unentschieden»; 5, 6 = "trifft zu». 


\section{Facharztprüfung}

Die Facharztprüfung wurde mehrheitlich positiv bewertet. Mehr als drei Viertel der Befragten gaben an, dass diejenigen Kompetenzen abgefragt wurden, die auch im Lernzielkatalog enthalten sind. Die Mehrheit fühlte sich an der Facharztprüfung fair behandelt und auch die Bewertung der Leistung entsprach ihren Erwartungen. Die Ergebnisse zur Beurteilung der Facharztprüfung sind in Tabelle 2 dargestellt.

Bei der Beurteilung der Facharztprüfung zeigen sich aber auch fachspezifische Unterschiede. Die Frage, ob nur diejenigen Kompetenzen abgefragt wurden, die auch im Lernzielkatalog enthalten sind, wurde von den Chirurgen am häufigsten bejaht. In der Anästhesiologie sowie der Orthopädischen Chirurgie und Traumatologie des Bewegungsapparates wurde diese Frage hingegen von mehr als einem Drittel der Befragten verneint. Auffallend ist, dass bei der Psychiatrie und
Psychotherapie jeder vierte Befragte angab, dass die Bewertung der Facharztprüfung nicht den Erwartungen entsprochen hätte.

\section{Allgemeine Zufriedenheit mit der Weiterbil- dung zum Facharzt}

Es zeigt sich, dass die Facharztabsolvent(inn)en mehrheitlich zufrieden sind mit der Qualität der Weiterbildung. Das gleiche Bild zeigt sich auch bei der Vorbereitung auf den Berufsalltag. Die Mehrheit der Befragten fühlte sich gut auf den Berufsalltag vorbereitet. Auffallend ist aber, dass weniger als die Hälfte der Befragten das Gefühl hatte, von ihren Weiterbildungsverantwortlichen immer ausreichend Unterstützung erfahren zu haben. Die Ergebnisse sind in Tabelle 3 aufgeführt. Die Antworten wurden für eine übersichtlichere Darstellung in drei Kategorien zusammengefasst: trifft nicht zu (1-2), unentschieden (3-4), trifft zu (5-6).

Tabelle 2: Beurteilung der Facharztprüfung

\begin{tabular}{|c|c|c|c|c|c|c|}
\hline & \multicolumn{2}{|c|}{$\begin{array}{l}\text { Wurden in der Facharzt- } \\
\text { prüfung nur diejenigen } \\
\text { Kompetenzen abgefragt, } \\
\text { die auch im Lernzielkata- } \\
\text { log enthalten sind? }\end{array}$} & \multicolumn{2}{|c|}{$\begin{array}{l}\text { Fühlten Sie sich in } \\
\text { der Facharztprüfung fair } \\
\text { behandelt? }\end{array}$} & \multicolumn{2}{|c|}{$\begin{array}{l}\text { Entsprach die Bewertung } \\
\text { lhrer Leistung an } \\
\text { der Facharztprüfung Ihren } \\
\text { Erwartungen? }\end{array}$} \\
\hline & ja & nein & ja & nein & ja & nein \\
\hline Gesamt (N = 1623) & $77 \%$ & $23 \%$ & $90 \%$ & $10 \%$ & $86 \%$ & $14 \%$ \\
\hline Chirurgie $(n=67)$ & $88 \%$ & $12 \%$ & $85 \%$ & $15 \%$ & $82 \%$ & $18 \%$ \\
\hline Gynäkologie ( $n=67$ ) & $76 \%$ & $24 \%$ & $88 \%$ & $12 \%$ & $92 \%$ & $8 \%$ \\
\hline Kinder- und Jugendmedizin $(n=103)$ & $78 \%$ & $22 \%$ & $90 \%$ & $10 \%$ & $89 \%$ & $12 \%$ \\
\hline Psychiatrie ( $n=172$ ) & $71 \%$ & $29 \%$ & $83 \%$ & $17 \%$ & $73 \%$ & $27 \%$ \\
\hline
\end{tabular}

Anmerkung: Frage: «Bitte beantworten Sie die folgenden Fragen zu Ihrer Facharztprüfung.»

Tabelle 3: Beurteilung der allgemeinen Zufriedenheit mit der Weiterbildung zum Facharzt.

\begin{tabular}{|c|c|c|c|c|c|c|c|c|c|}
\hline & \multicolumn{3}{|c|}{$\begin{array}{l}\text { Ich war nach meiner Zeit } \\
\text { als Arzt in Weiterbildung } \\
\text { gut für den Berufsalltag } \\
\text { als Facharzt vorbereitet. }\end{array}$} & \multicolumn{3}{|c|}{$\begin{array}{l}\text { Ich habe von meinen } \\
\text { Weiterbildungsverantwort- } \\
\text { lichen immer ausreichend } \\
\text { Unterstützung erfahren. }\end{array}$} & \multicolumn{3}{|c|}{$\begin{array}{l}\text { Ich bin insgesamt zufrie- } \\
\text { den mit der Qualität } \\
\text { meiner Weiterbildung } \\
\text { zum Facharzt. }\end{array}$} \\
\hline Gesamt ( $=1623$ ) & $4 \%$ & $36 \%$ & $60 \%$ & $11 \%$ & $42 \%$ & $46 \%$ & $4 \%$ & $36 \%$ & $60 \%$ \\
\hline Chirurgie $(n=67)$ & $5 \%$ & $36 \%$ & $60 \%$ & $8 \%$ & $51 \%$ & $42 \%$ & $8 \%$ & $39 \%$ & $53 \%$ \\
\hline Gynäkologie ( $n=67$ ) & $3 \%$ & $30 \%$ & $67 \%$ & $13 \%$ & $43 \%$ & $43 \%$ & $3 \%$ & $49 \%$ & $48 \%$ \\
\hline Kinder- und Jugendmedizin $(n=103)$ & $6 \%$ & $38 \%$ & $56 \%$ & $9 \%$ & $47 \%$ & $45 \%$ & $4 \%$ & $34 \%$ & $62 \%$ \\
\hline Psychiatrie $(n=172)$ & $3 \%$ & $33 \%$ & $65 \%$ & $15 \%$ & $43 \%$ & $42 \%$ & $4 \%$ & $37 \%$ & $59 \%$ \\
\hline
\end{tabular}

Anmerkung: Frage: «Inwiefern treffen die folgenden Aussagen in Bezug auf die Weiterbildung zum Facharzt auf Sie zu?» Die Antwortskala reichte von 1 "trifft überhaupt nicht zu» bis 6 "trifft voll und ganz zu». Für die Auswertungen wurden die Antworten wie folgt zusammengefasst: 1, 2 = "trifft nicht zu»; 3, 4 = «unentschieden; 5, 6 = «trifft zu». 


\section{Diskussion}

Die vorliegende Befragung liefert ein umfassendes Bild darüber, wie die Fachärzte und Fachärztinnen ihre Weiterbildung im Nachhinein einschätzen. Erfreulich ist, dass sie insgesamt klar positiv beurteilt wird. Die meisten Befragten haben das Gefühl, gut auf die Arbeit als Facharzt vorbereitet worden zu sein. Die Ergebnisse zeigen aber auch, dass gewisse Fachkompetenzen nicht in dem Umfang erlernt werden konnten, wie er im Hinblick auf die späteren beruflichen Anforderungen als notwendig empfunden wird. Dazu gehören die Gesprächsführung, die Beurteilung der Arbeitsfähigkeit, die ökonomischen Grundlagen, der Umgang mit Fehlern und auch das Erlernen von Führungsfunktionen. Besonders die Gesprächsführung und die Beurteilung der Arbeitsfähigkeit sind Kompetenzen, die für die praktische Tätigkeit als wichtig bis sehr wichtig eingestuft werden. Die Vermittlung dieser Kompetenzen während der Weiterbildung wird aber als ungenügend oder knapp genügend beurteilt, wobei es zwischen den verschiedenen Fachrichtungen deutliche Unterschiede gibt. Es ist bemerkenswert, dass die Defizite kaum bei den fachspezifischen Lernzielen, sondern vor allem bei den allgemeinen Lernzielen der ärztlichen Tätigkeit lokalisiert werden.

Die Umfrageergebnisse weisen auch darauf hin, dass in Bezug auf das Betreuungsverhältnis aus der Sicht der ehemaligen Assistenzärzte ein Verbesserungsbedürfnis besteht. Weniger als die Hälfte der Befragten ist nämlich der Meinung, während der Weiterbildung wirklich ausreichend Unterstützung seitens der Weiterbildungsverantwortlichen erfahren zu haben. Inwieweit diese Aussage objektivierbar ist und was die Gründe dafür sein könnten, bedarf sicher einer detaillierteren Analyse.

Die Lernzielkataloge wurden insgesamt als angemessen beurteilt. Die Befragten sind also der Meinung, dass die Lernziele eine gute Grundlage für die selbständige Berufstätigkeit bilden. Ausnahmen finden sich vor allem in Fachrichtungen wie der Chirurgie oder der Orthopädischen Chirurgie, wo nicht nur von Einzelnen die Meinung vertreten wird, dass im Lernzielkatalog wichtige Lernziele fehlen. Auch zur Strukturierung des vorgesehenen Weiterbildungsablaufs stehen Fragen im Raum. Die Ergebnisse weisen darauf hin, dass es für verschiedene Disziplinen wünschenswert wäre, ihren Lernzielkatalog und ihre Weiterbildungskonzepte zu überprüfen.

Die Facharztprüfung wird insgesamt als fair wahrgenommen. In der Anästhesiologie und der orthopädischen Chirurgie hatte jeder dritte Befragte den Eindruck, er sei über Kompetenzen abgefragt worden, welche nicht im Lernzielkatalog enthalten seien. Aufgrund der Daten lässt sich nicht beurteilen, ob die Lernziele nicht eindeutig genug beschrieben sind oder ob tatsächlich Kompetenzen abgefragt werden, welche im Lernzielkatalog fehlen.

Wenn die hier präsentierten Ergebnisse in den zuständigen Gremien analysiert und zum Anlass für Anpassungen genommen werden, hat diese Umfrage ihren Zweck erfüllt. Auch wenn die Weiterbildung in der individuellen Rückschau insgesamt positiv beurteilt wird, ist es wohl unvermeidlich, dass immer wieder einzelne Defizite festgestellt werden, die Anlass für Verbesserungen und Optimierungen geben können.

\section{Anmerkung}

Ein Foliensatz mit einer umfassenden Beschreibung der Studienergebnisse kann unter der folgenden E-Mail-Adresse angefordert werden: cb-projekt-fmh[at]ethz.ch

\section{Literatur}

1 Siegrist M, Gutscher H, Giger M. Was haben Betriebskultur, Motivation und Fremdjahr mit der Weiterbildung zu tun? Schweiz Ärztezeitung 2004; 85(15):783-91.

2 Cantor JC, Baker LC, Hughes RG. Preparedness for practice: young physicians' views of their professional education. Jama. 1993;270:1035-40.

3 Lieberman L, Hilliard, RI. How well do paediatric residency programmes prepare residents for clinical practice and their future careers? Med Educ. 2006;40:539-46.

4 van der Horst K, Siegrist M, Orlow P, Giger M. Residents' reasons for specialty choice: influence of gender, time and career. Med Educ. 2010;44:595-602. 